\title{
Performance Analysis of Regenerative Feed Heating in A Steam Power Plant
}

\author{
Ch.vijaya $\operatorname{kumar}^{1}$ M.tech(cad/cam),Dhananjaya umar $^{2}$ M.E \\ ${ }^{1,2}$ Mechanical,Gnit/Jntuh,India
}

\begin{abstract}
The development of any country relates on capital energy consumption. The demand for power generation on the large scale is increasing day by day. Owing to their major contribution towards power production thermal plant shave vital role to play in the development of nation due to the scarcity of power, every power plant needs to be operated at maximum level of efficiency. The feed water heaters from a part of the regenerative system to increase the overall thermal efficiency of the plant In the present world, that parameter which directly or indirectly influences the performance of a heater has been studied the factor such as inlet temperature, saturation temperature, terminal tap difference, drain cool approach \& Temperature raise are studied The data for the performance of a test and the subsequent calculations are collected from HP heaters of Unit 1 VTPS(Vijayawada Thermal Power Station)
\end{abstract}

Keywords: Feed water, power station, Regenerative, Thermal, turbines, operational cost

\section{Introduction}

The Regenerative Cycle starts from turbine. The regenerative feed heating system cycle starts from the condenser at L.P end and ends at economizer inlet H.P end. Various auxiliaries like condenser, pump, main ejectors, gland steam condenser, drain cooler, H.P heaters etc. are situated in the system. The L.P heaters drain cooler, main ejectors, and gland steam condenser are located in the condensate cycle between condensate pump $\&$ in the deaerator. The H.P heaters are located in the feed water cycle between deaerator and the boiler. The six extractions from the turbine are provided to 3 horizontal L.P heaters, deaerator and 2 Horizontal H.P heaters.

\subsection{Functions of extraction Drain System:}

The turbine extraction lines are connected to extraction drain points to drain condensate during warming up of pipes, during heater out condition and during turbine drip operation. The function of L.P heater is to increase the temperature of the feed water condensate discharged from drain cooler. The function of H.P heater to increase the temperature of the drain to the maximum desired required range. The function of deaerator is the dissolved gases like oxygen, $\mathrm{CO} 2, \mathrm{NH} 2$ which are harmful and present in feed water, are eliminated and heats the incoming feed water \& Acts as a reservoir to provide a sudden and instantaneous demand.

\subsection{Advantages Of Regenerative Feed Heating System:}

It improves the cycle efficiency. The metal temperature of boiler tube reduces by taking feed heaters in service and hence increases the boiler tube life and reduces the outage due to tube leakages. Extractions from last stages of turbine also act as moisture extractor and hence reduce the blade damage due to water droplets impact. Cost per unit of electrical power reduces by using with feed heaters.

\subsection{Disadvantages Of Regenerative Feed Heating System:}

Increase cost of pipe frame work and also increase mechanical losses with the some steam input less work is achieved. Strength of turbine decreases due to the holes provided for extraction of steam from different stages. The temperature of the flue gases may reach to dew point temperature Decreases the extent of heat extraction from the gases economizer, Regenerative feed heating involves the process of improving the efficiencies of turbine and to produce move work.

\subsection{Basic feed heating system:}

For the basic feed heating system for each process in vapor power cycle. it is possible to assume a hypothetical (or)ideal process which represents the basic intended operation. For basic feed system we used Rankin cycle in this when all these four processes are ideal, the cycle is an ideal cycle called Rankin cycle. For the purpose if analysis, the Rankin cycle is assumed to be carried out in a steady flow operation, applying steady flow engineering equation (SFEE) to each of the process on the basis of the process on the basis of unit mass of fluid, and neglecting changes in kinetic and potential energy. The work and heat quantities can be evaluated in terms of the properties of the fluid. Usually, the pump work is small compared to the turbine work, and is sometimes neglected then $\mathrm{h}_{4}=\mathrm{h}_{3}$ and the cycle efficiency approximately becomes 
$\eta=\frac{\mathrm{h} 1-\mathrm{h} 2}{\mathrm{~h} 1-\mathrm{h} 4}$

In the direction opposite to the steam flow. In turbine, the steam is thus headed before entering into the boiler; such a system is known as Regenerative heating. The ideal regenerative cycle has efficiency equal to that efficiency of Carnot cycle with same heat supply and heat rejection temperatures.

All the heat added from an external source $\left(\theta_{1}\right)$ is at constant temperature $\left(T_{1}\right)$, and all the heat rejected $\left(\theta_{2}\right)$ is at the constant temperature $\left(\mathrm{T}_{2}\right)$ both being reversible then

$\theta_{1}=\mathrm{h}_{1}-\mathrm{h}_{4}{ }^{\mathrm{I}}=\mathrm{T}_{1}\left(\mathrm{~s}_{1}-\mathrm{s}_{4}\right)$

$\theta_{2}=\mathrm{h}_{2}-\mathrm{h}_{3}=\mathrm{T}_{2}\left(\mathrm{~s}_{2}-\mathrm{s}_{3}\right)$

$\mathrm{S} 4-\mathrm{s} 3=\mathrm{s}_{1}-\mathrm{S}_{2}$

$\mathrm{S} 1-\mathrm{s} 4=\mathrm{s}_{2}-\mathrm{S}_{3}$

$?=1-\theta_{2} / \theta_{1}=1-\mathrm{T}_{2} / \mathrm{T}$

The efficiency of the ideal regenerative cycle is equal to Carnot cycle efficiency writing the steady flow energy equation for the turbine.

$\mathrm{H}_{1}-\mathrm{W}_{\mathrm{t}}-\mathrm{h}_{2}+\mathrm{h}_{4}-\mathrm{h}_{4}=0$

$\mathrm{W}_{\mathrm{t}}=\left(\mathrm{h}_{1}-\mathrm{h}_{2}\right)-\left(\mathrm{h}_{4}-\mathrm{h}_{4}\right)$

The pump work remains the same as in the Rankin cycle

$\mathrm{W}_{\mathrm{p}}=\mathrm{h}_{4}-\mathrm{h}_{3}$

The network output of the ideal regenerate cycle is thus less, and hence its steam rate will be move. Although it is more efficient when compared with the Rankin cycle

\section{1regenerative Cycle With Single Feed Water Heater:}

II. Regenerative Cycles With Feed Water Heaters:

The steam at $\mathrm{p}$, pressure enters the turbine at point $\mathrm{A}$. let a small amount of wet steam of $\mathrm{m} \mathrm{Kg}$ after partial expansion at pressure $\mathrm{p}_{2}$ be drained from turbine at point $\mathrm{B}$ and enters the feed water heater. The remaining steam at pressure $\mathrm{P}_{3}$ is further expanded in turbine and leaves at point "c" The steam is then condensed in the condenser. The condensate from the condenser is pumped into the feed water heater where it mixes up with steam extracted is just sufficient to cause the steam leaving the feed water to be saturated.

?cycle $=\frac{\mathrm{Wt}}{\mathrm{Q} 1}=\frac{(\mathrm{h} 1-\mathrm{h} 2)+(1-\mathrm{m})(\mathrm{h} 2-\mathrm{h} 3)}{(\mathrm{h} 1-\mathrm{h} 6)}$

$\mathrm{W}_{\mathrm{t}}=$ Turbine work

$\mathrm{h}_{1}=$ Enthalpy of steam entering the turbine at A

$\mathrm{h}_{2}=$ Enthalpy of bled steam

$\mathrm{h}_{3}=$ Enthalpy of steam leaving the turbine at $\mathrm{C}$

$\mathrm{h}_{\mathrm{f} 1}=$ sensible heat of feed water leaving the feed water heater.

$\mathrm{h}_{\mathrm{f} 3}=$ Enthalpy (or) sensible heat of steam leaving the condenser.

$\mathrm{m}=$ Amount of bled steam per $\mathrm{kg}$ of steam supplied

Where $\mathrm{m}=\frac{\mathrm{hf} 2-\mathrm{hf} 3}{\mathrm{~h} 2-\mathrm{hf} 3}$

Efficiency of cycle including the effect of bleeding $=\frac{\text { Total work done }}{\text { total heat supplied }}=\frac{(\mathrm{h} 1-\mathrm{h} 2)+(1-\mathrm{m})(\mathrm{h} 2-\mathrm{h} 3)}{(\mathrm{h} 1-\mathrm{h} 2)}$

\subsection{Regenerative Cycle with Two Feed Water Heaters:}

The steam at pressure $p_{1}$ enters the turbine at point " $A$ " Let a small amount of steam after partial expansion $\mathrm{p}_{2}$ be drained from the turbine at point $\mathrm{B}_{1}$ enters the feed water heater 2.the remaining steam equal $\mathrm{t}$ $\left(1-\mathrm{m}_{1}-\mathrm{m}_{2}\right) \mathrm{Kg}$ is further expanded in the turbine and leaves it a point "c". The steam is then condensed in the condenser, The condensate from the condenser is pumped in to the feed water where it mixes with the steam extracted from the turbine.

Let us consider $1 \mathrm{Kg}$ steam

$\mathrm{h}_{1}=$ Enthalpy of steam entering the turbine at A

$\mathrm{h}_{2}=$ Enthalpy of steam bled at B to L.P heater

$\mathrm{h}_{3}=$ Enthalpy of steam bled at $\mathrm{B}_{1}$ to H.P heater

$\mathrm{h}_{4}=$ Enthalpy of steam leaving the turbine at $\mathrm{C}$

$\mathrm{h}_{\mathrm{f} 2}=$ Enthalpy of feed water leaving the feed water heater 1

$\mathrm{h}_{\mathrm{f} 3}=$ Enthalpy of feed water leaving the feed water heater2

$\mathrm{h}_{\mathrm{f} 4}=$ Enthalpy of steam leaving the condenser.

$\mathrm{m}_{1}=$ Amount of steam bled at B per $\mathrm{kg}$ of steam supplied

$\mathrm{m}_{2}=$ Amount of steam bled at $\mathrm{B}_{1}$ per $\mathrm{kg}$ of steam supplied

Here $\mathrm{m}_{1}=\frac{\mathrm{hf} 2-\mathrm{hf} 3}{\mathrm{~h} 2-\mathrm{hf} 3}$

$\mathrm{m}_{2}=\frac{(1-\mathrm{m} 1)(\mathrm{hf} 3-\mathrm{hf} 4)}{\mathrm{h} 3-\mathrm{hf} 4}$ 
Function of Extraction Drain System:-

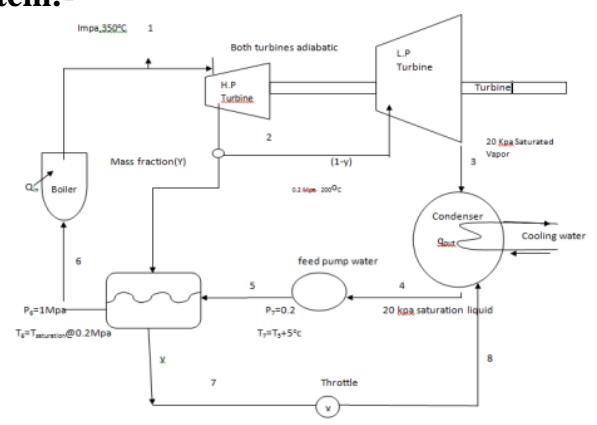

Turbine Extraction System:-

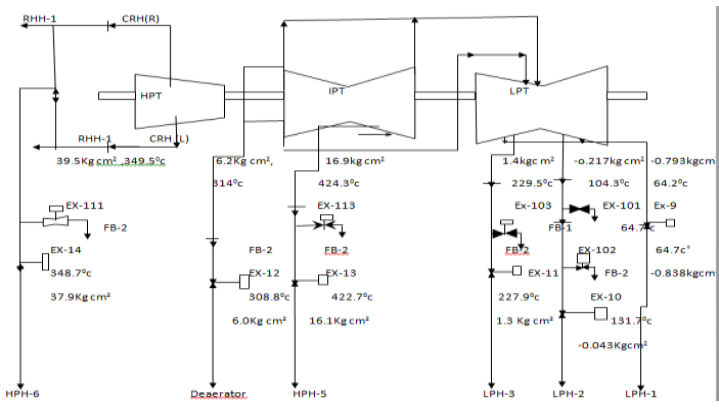

III. Technical Data:

HPH5:Vertical coil type with integral de superheating and drain cooling zones

\begin{tabular}{|c|c|c|}
\hline Quality of extraction steam & $\mathrm{Kg} / \mathrm{hr}$ & 17661 \\
\hline Pressure of extraction steam(max) & ata & 13.16 \\
\hline Enthalpy of extraction steam & Kcal/kg & 802.84 \\
\hline Qty. of feed water & t/hr & 662 \\
\hline Temp. of feed water at inlet & ${ }^{\circ} \mathrm{C}$ & 169.3 \\
\hline Temp. of feed water at outlet & ${ }^{\circ} \mathbf{C}$ & 186.0 \\
\hline Size of tubes(D.S.Zone) & $\mathbf{m m}$ & $32 \times 4.0$ \\
\hline No .of spiral coils in de-superheating zone & ............ & 32 \\
\hline $\begin{array}{l}\text { Surface area: } \\
\text { De-superheating zone: } \\
\text { Condensating zone: } \\
\text { Drain cooling zone: }\end{array}$ & $\begin{array}{l}\mathrm{Mm}^{2} \\
\mathrm{Mm}^{2} \\
\mathrm{Mm}^{2}\end{array}$ & $\begin{array}{l}84.5 \\
602 \\
84.5\end{array}$ \\
\hline Design pressure on shell side & atg & 14 \\
\hline Design pressure on water side & atg & 265 \\
\hline Design pressure on shell side & ${ }^{\circ} \mathbf{C}$ & 210 \\
\hline Design pressure on water side & ${ }^{\circ} \mathrm{C}$ & 210 \\
\hline $\begin{array}{l}\text { Test pressure on the shell side } \\
\text { Test pressure on water side }\end{array}$ & $\begin{array}{l}\text { atg } \\
\text { atg }\end{array}$ & $\begin{array}{l}21 \\
400\end{array}$ \\
\hline Overall height & $\mathbf{m m}$ & 9625 \\
\hline
\end{tabular}

Technical Data:

HPH6:Vertical coil type with integral de superheating and drain cooling zones

\begin{tabular}{|l|l|l|}
\hline Quality of extraction steam & $\mathrm{Kg} / \mathrm{hr}$ & $\mathbf{4 7 2 7 8}$ \\
\hline Pressure of extraction steam(max) & $\mathrm{ata}$ & $\mathbf{2 8 . 5 3}$ \\
\hline Enthalpy of extraction steam & $\mathrm{Kcal} / \mathrm{kg}$ & $\mathbf{7 3 3 . 6 7}$ \\
\hline Qty. of feed water & $\mathrm{t} / \mathrm{hr}$ & $\mathbf{6 6 2}$ \\
\hline Temp. of feed water at inlet & ${ }^{\circ} \mathrm{C}$ & $\mathbf{1 8 6}$ \\
\hline Temp. of feed water at outlet & ${ }^{\circ} \mathrm{C}$ & $\mathbf{2 2 2 . 3}$ \\
\hline Size of tubes(D.S.Zone) & $\mathrm{mm}$ & $\mathbf{O D ~ 3 2 x} 4$ \\
\hline No .of spiral coils in de-superheating zone & $\ldots \ldots \ldots . .$. & $\mathbf{3 2}$ \\
\hline Surface area: & & \\
De-superheating zone: & $\mathrm{Mm}^{2}$ & $\mathbf{8 4 . 5}$ \\
Condensating zone: & $\mathrm{Mm}^{2}$ & $\mathbf{6 0 2}$ \\
Drain cooling zone: & $\mathrm{Mm}^{2}$ & $\mathbf{8 4 . 5}$ \\
\hline Design pressure on shell side & $\mathrm{atg}$ & $\mathbf{3 2}$ \\
\hline Design pressure on water side & $\mathrm{atg}$ & $\mathbf{2 6 5}$ \\
\hline Design pressure on shell side & ${ }^{\circ} \mathrm{C}$ & $\mathbf{2 5 0}$ \\
\hline Design pressure on water side & ${ }^{\circ} \mathrm{C}$ & $\mathbf{2 5 0}$ \\
\hline Test pressure on the shell side & $\mathrm{atg}$ & $\mathbf{4 8}$ \\
Test pressure on water side & $\mathrm{atg}$ & $\mathbf{4 0 0}$ \\
\hline Overall height & $\mathrm{mm}$ & $\mathbf{9 6 2 5}$ \\
\hline
\end{tabular}


Technical Data:

HPH7:Vertical coil type with integral de

\begin{tabular}{|c|c|c|}
\hline Quality of extraction steam & Kg/hr & 32441 \\
\hline Pressure of extraction steam(max) & ata & 42.83 \\
\hline Enthalpy of extraction steam & Kcal/kg & 756.68 \\
\hline Qty. of feed water & $\mathbf{t} / \mathbf{h r}$ & 662 \\
\hline Temp. of feed water at inlet & ${ }^{\circ} \mathrm{C}$ & 222.3 \\
\hline Temp. of feed water at outlet & ${ }^{\circ} \mathrm{C}$ & 245.7 \\
\hline Size of tubes(D.S.Zone) & $\mathbf{m m}$ & OD 32x4 \\
\hline No of spiral coils in de-superheating zone & ................ & 32 \\
\hline $\begin{array}{l}\text { Surface area: } \\
\text { De-superheating zone: } \\
\text { Condensating zone: } \\
\text { Drain cooling zone: }\end{array}$ & $\begin{array}{l}\mathbf{M m}^{2} \\
\mathbf{M m}^{2} \\
\mathbf{M m}^{2}\end{array}$ & $\begin{array}{l}84.5 \\
602 \\
84.5 \\
\end{array}$ \\
\hline Design pressure on shell side & atg & 32 \\
\hline Design pressure on water side & atg & 265 \\
\hline Design pressure on shell side & ${ }^{\circ} \mathrm{C}$ & 250 \\
\hline Design pressure on water side & ${ }^{\circ} \mathrm{C}$ & 250 \\
\hline $\begin{array}{l}\text { Test pressure on the shell side } \\
\text { Test pressure on water side }\end{array}$ & $\begin{array}{l}\text { atg } \\
\text { atg }\end{array}$ & $\begin{array}{l}48 \\
400\end{array}$ \\
\hline Overall height & $\mathbf{m m}$ & 9625 \\
\hline
\end{tabular}

Superheating and drain cooling zones

\begin{tabular}{|c|c|c|c|c|c|}
\hline S.no & & & & & \\
\hline 1 & NO. Of heaters & ................ & 3 & & \\
\hline 2 & Type & .................... & Vertical & & \\
\hline 3 & HP HEATERS & & HPH5 & НРН6 & HPH7 \\
\hline 4 & Inlet FW flow & t/hr & 662 & 662 & 662 \\
\hline 5 & FW inlet temp & ${ }^{\circ} \mathbf{C}$ & 169.3 & 184.8 & 221.73 \\
\hline 6 & FW outlet temp & ${ }^{\circ} \mathrm{C}$ & 184.8 & 221.73 & 244.6 \\
\hline 7 & EXtern.Steam flow & t/hrs & 17.66 & 41.28 & 32.44 \\
\hline 8 & Steam(turbine end) & Ata & 13.15 & 28.853 & 42.83 \\
\hline 9 & $\begin{array}{l}\text { Steam } \\
\text { end) }\end{array} \quad$ temp(turbine & ${ }^{\circ} \mathbf{C}$ & 453 & 330 & 383 \\
\hline 10 & TTD & ${ }^{\circ} \mathrm{C}$ & 2.0 & 4.5 & 2.5 \\
\hline 11 & DCA & ${ }^{\circ} \mathrm{C}$ & 10 & 15 & 7.5 \\
\hline 12 & TR & ${ }^{\circ} \mathrm{C}$ & ........... & ............ & ............... \\
\hline
\end{tabular}

Design Data:

Test Data:

\begin{tabular}{|c|c|c|c|c|c|}
\hline S.NO. & Description & Units & Run 1 & Run 2 & Run 3 \\
\hline 1 & Load & MW & 209 & 211 & 209 \\
\hline 2 & Ms pressure & $\mathrm{Kg} / \mathrm{cm}^{2}$ & 128.98 & 118.8 & 129.02 \\
\hline 3 & Fw flow & t/hr & 687.10 & 701.97 & 684.92 \\
\hline 4 & HPH5 shell pressure & $\mathrm{Kg} / \mathrm{cm}^{2}$ & 11.41 & 11.61 & 11.32 \\
\hline 5 & HPH6 shell pressure & $\mathrm{Kg} / \mathrm{cm}^{2}$ & 25.01 & 25.50 & 25.08 \\
\hline 6 & HPH7 shell pressure & $\mathrm{Kg} / \mathrm{cm}^{2}$ & 39.02 & 39.87 & 39.04 \\
\hline 7 & FW temp HPH5 IN & ${ }^{\circ} \mathrm{C}$ & 162.52 & 161.95 & 162.45 \\
\hline 8 & FW temp HPH5 OUT & ${ }^{\circ} \mathrm{C}$ & 179.01 & 179.01 & $\mathbf{1 7 8 . 7 8}$ \\
\hline 9 & FW temp HPH6 OUT & ${ }^{\circ} \mathrm{C}$ & 220.68 & 221.58 & 221.05 \\
\hline 10 & FW temp HPH7 OUT & ${ }^{\circ} \mathrm{C}$ & 245.32 & 246.34 & 245.48 \\
\hline 11 & Drain temp HPH5 & ${ }^{\circ} \mathrm{C}$ & 183.07 & 210.29 & 235.1 \\
\hline 12 & Drain temp HPH6 & ${ }^{\circ} \mathrm{C}$ & 183.51 & 210.95 & 236.01 \\
\hline 13 & Drain temp HPH7 & ${ }^{\circ} \mathrm{C}$ & $\mathbf{1 8 2 . 7 6}$ & 210.38 & 235.25 \\
\hline
\end{tabular}

The extract from the test data (Run-1) is consolidated in the table given below:

\begin{tabular}{|c|c|c|c|c|c|}
\hline $\begin{array}{l}\text { S. } \\
\text { No. }\end{array}$ & Description & Design $^{\circ} \mathrm{C}$ & Design $^{\circ} \mathrm{C}$ & Deviation $^{\circ} \mathrm{C}$ & $\begin{array}{l}\text { HR deviation } \\
\text { Kcal/KWh }\end{array}$ \\
\hline 1 & HPH7 TTD & 2.5 & 2.2 & -0.3 & -0.57 \\
\hline 2 & HPH7 DCA & 7.5 & 14.2 & 6.7 & 1.6 \\
\hline 3 & HPH7 TR & ...... & ...... & ..... & ....... \\
\hline 4 & HPH6 TTD & 4.5 & 4.4 & -0.1 & -0.19 \\
\hline 5 & HPH6 DCA & 15 & 31.3 & 16.3 & 3.89 \\
\hline 6 & HPH6 TR & ...... & ...... & ........ & ....... \\
\hline 7 & HPH5 TTD & 2.0 & 8.7 & 6.7 & 12.78 \\
\hline 8 & HPH5 DCA & 10 & 20.5 & 10.5 & 2.51 \\
\hline 9 & HPH5 TR & ......... & .......... & ......... & ......... \\
\hline
\end{tabular}




\section{Calculation\& Analysis:}

1) Terminal Temperature Difference (TTD):

TTD $=$ T sat $-\mathbf{T}(\mathbf{f w}$ out $)$

- Where, $\mathbf{T}($ sat $)=$ Saturation temp taken at the heater shell pressure, ${ }^{\circ} \mathrm{C}$

$\mathrm{T}(\mathrm{fw}$ out $)=$ Temperature of feed water leaving the heater, ${ }^{\circ} \mathrm{C}$

2) Drain cooling approach (DCA):

DCA $=\mathbf{T}$ (drain temp) $-\mathbf{T}$ (feed water entering)

Where, $\mathrm{T}$ (drain) $=$ temp of the drain leaving the heater, ${ }^{\circ} \mathrm{C}$

$\mathrm{T}\left(\mathrm{fw}\right.$ in) $=$ Temperature of feed water entering the heater, ${ }^{\circ} \mathrm{C}$

3) Temp rise across heater (TR):

$\mathbf{T R}=\mathbf{T}$ (feed water entering) $-\mathbf{T}($ feed water leaving)

Model calculations -1 For HPH- 7:

Pressure $=39.02$ bar

T sat $=248.029{ }^{\circ} \mathrm{C}$ (from stem tables)

T f w out $=245.32{ }^{\circ} \mathrm{C}$

TTD $=248.029-245.32=2.71^{\circ} \mathrm{C}$

$\mathrm{T}$ drain $=235.10{ }^{\circ} \mathrm{C}$

$\mathrm{T}$ fw in $=220.88{ }^{\circ} \mathrm{C}$

DCA $=235.10-220.88=14.22{ }^{\circ} \mathrm{C}$

T fw out $=245.32{ }^{\circ} \mathrm{C}$

$\mathrm{T} f \mathrm{w}$ in $=245.32-220.88=24.4$

Model calculations -1 For HPH - 6:

Pressure $=25.01 \mathrm{bar}$

T sat $=223.97{ }^{\circ} \mathrm{C}$ (from stem tables)

$\mathrm{T}$ fw out $=211.88^{\circ} \mathrm{C}$

$$
=3.1^{\circ} \mathrm{C}
$$

TTD $=223.97-211.88=3.1^{\circ} \mathrm{C}$

$\mathrm{T}$ drain $=210.29^{\circ} \mathrm{C}$

$\mathrm{T}$ fw in $=179.01{ }^{\circ} \mathrm{C}$

DCA $=210.29-179.01=31.28{ }^{\circ} \mathrm{C}$

$\mathrm{T}$ fw out $=210.88{ }^{\circ} \mathrm{C}$

$\mathrm{T}$ fw in $=179-01^{\circ} \mathrm{C}$

$\mathrm{TR}=210.88-179.01=41.87^{\circ} \mathrm{C}$

Model calculations -1 For HPH - 5:

Pressure $=11.4$ bar

T sat $=187.68{ }^{\circ} \mathrm{C}$ (from stem tables)

$\mathrm{T}$ fw out $=179.01{ }^{\circ} \mathrm{C}$

TTD $=187.68-179.01=8.67^{\circ} \mathrm{C}$

Model calculation : 2

Using the amount of heat lost by the feed water and the amount of heat gained by the steam the performance of the HP heaters can be analyzed. it is know that the amount of heat gained should be equal to the amount of heat lost but the case being heaters or any other mechanical components the heat transfer is not that much perfect as it is effected by many losses.

Model calculations -2 For HPH - 7:

External steam flow $=32 \mathrm{t} / \mathrm{hr}$

Steam pressure $=37.5 \mathrm{bar}$

Steam temperature $=383{ }^{\circ} \mathrm{C}$

Drain pressure $=37.5 \mathrm{bar}$

Drain temperature $=235^{\circ} \mathrm{C}$

Using mollies diagram,

The enthalpy of steam is h steam $=3170 \mathrm{KJ} / \mathrm{kg}$

The enthalpy of drain is h drain $=2820 \mathrm{KJ} / \mathrm{kg}$ 
h steam $-\mathrm{h}$ drain $=3170-02820=350 \mathrm{KJ} / \mathrm{kg}$

Amount of heat lost $=$ flow $\mathrm{x}$ enthalpy

$$
\begin{aligned}
& =32 \times 350 \times 10^{3} \mathrm{KJ} / \mathrm{hr} \\
& =11200 \times 10^{3} \mathrm{~kJ} / \mathrm{hr}
\end{aligned}
$$

As there is under cooling, the amount of heat lost in the drain is $=\mathrm{m} \mathrm{cp}$ ( $\mathrm{T}$ sat $-\mathrm{T}$ drain)

$=662 \times 1 \times(246-235)$

Total amount of heat lost $=(911200+7282) \times 10^{3}$

$$
=7282 \times 10^{3} \mathrm{~kJ} / \mathrm{kg}
$$

$$
\begin{aligned}
& =662 \times 1 \times(245-221) \\
& =15888 \times 10^{3} \mathrm{~kJ} / \mathrm{hr}
\end{aligned}
$$

Model calculations -2 For HPH - 6:

External steam flow $=41 \mathrm{t} / \mathrm{hr}$

Steam pressure $=21$ bar

Steam temperature $=330{ }^{\circ} \mathrm{C}$

Drain pressure $=25$ bar

Drain temperature $=210^{\circ} \mathrm{C}$

Drain flow $=73 \mathrm{t} / \mathrm{hr}$

Using mollies diagram,

The enthalpy of steam is h steam $=3070 \mathrm{~kJ} / \mathrm{kg}$

The enthalpy of drain is h drain $=2825 \mathrm{~kJ} / \mathrm{kg}$

The enthalpy of drain leaving heater $\mathrm{H}$ drain $=3170 \mathrm{~kJ} / \mathrm{kg}$

$\mathrm{H}$ eat lost by steam $(\mathrm{Q} 1)=$ flow $\mathrm{x}$ enthalpy

$$
=41 \times 3070=125870 \times 10^{3}
$$

Heat lost by the drain leaving heater $7(\mathrm{Q} 2)=32 \times 3170$

Heat lost by drain $(\mathrm{Q} 3)=73 \times 2825$

$$
=101400 \times 10^{3} \mathrm{~kJ} / \mathrm{hr}
$$

Heat lost $=(\mathrm{Q} 1+\mathrm{Q} 2)-\mathrm{Q} 3$

$$
=2062258 \times 10^{3} \mathrm{~kJ} / \mathrm{hr}
$$

$$
\begin{aligned}
& =(125870+101400)-206225) \times 10^{3} \mathrm{~kJ} / \mathrm{hr} \\
& =21085 \times 10^{3} \mathrm{~kJ} / \mathrm{hr}
\end{aligned}
$$

Due to under cooling heat lost in drain $=\mathrm{m} \times \mathrm{Cp} \times(\mathrm{T}$ sat $-\mathrm{T}$ drain $)$

Total heat lost $=(21085+9268) \times 10^{3} \mathrm{~kJ} / \mathrm{hr}$

$$
\begin{aligned}
& =662 \times 1 \times(224-210) \\
& =9268 \times 10^{3} \mathrm{~kJ} / \mathrm{hr}
\end{aligned}
$$

$$
=30353 \times 10^{3} \mathrm{~kJ} / \mathrm{hr}
$$

Heat gain by the feed water $=\mathrm{m} \times \mathrm{Cp} \times($ Fwo $-\mathrm{F}$ win $)$

$$
\begin{aligned}
& =662 \times 1 \times(221-179) \\
& =27084 \times 10^{3} \mathrm{~kJ} / \mathrm{hr}
\end{aligned}
$$

Model calculations -2 For HPH - 5:

External steam flow $=17 \mathrm{t} / \mathrm{hr}$

Steam pressure $=11.5 \mathrm{bar}$

Steam temperature $=453{ }^{\circ} \mathrm{C}$

Drain pressure $=11.5 \mathrm{bar}$

Drain temperature $=183^{\circ} \mathrm{C}$

Drain flow $=90 \mathrm{t} / \mathrm{hr}$

Using mollies diagram,

The enthalpy of steam is h steam $=3380 \mathrm{~kJ} / \mathrm{kg}$

The enthalpy of drain is h drain $=2780 \mathrm{~kJ} / \mathrm{kg}$

The enthalpy of drain coming from heater $6 \mathrm{H}$ drain $=2825 \mathrm{~kJ} / \mathrm{kg}$

H eat lost by steam $(\mathrm{Q} 1)=$ flow $\mathrm{x}$ enthalpy

$=17 \times 3380=57460 \times 10^{3}$

Heat lost by the drain leaving heater $7(\mathrm{Q} 2)=73 \times 2825$

$=206225 \times 10^{3} \mathrm{~kJ} / \mathrm{hr}$

Heat lost by drain $(\mathrm{Q} 3)=90 \times 2780$ 
Heat lost $=(\mathrm{Q} 1+\mathrm{Q} 2)-\mathrm{Q} 3$

$$
=250200 \times 10^{3} \mathrm{~kJ} / \mathrm{hr}
$$

$$
\begin{aligned}
& =(57460+206225)-250200) \times 10^{3} \mathrm{~kJ} / \mathrm{hr} \\
& =13485 \times 10^{3} \mathrm{~kJ} / \mathrm{hr}
\end{aligned}
$$

Due to under cooling heat lost in drain $=\mathrm{m} \times \mathrm{Cp} \times(\mathrm{T}$ sat $-\mathrm{T}$ drain $)$

$$
\begin{aligned}
& =662 \times 1 \times(186-183) \\
& =1986 \times 10^{3} \mathrm{~kJ} / \mathrm{hr}
\end{aligned}
$$

where $\mathrm{m}=$ mass flow rate of steam

$\mathrm{cp}=1.000 \mathrm{~kJ} / \mathrm{kg}-\mathrm{k}$

\section{Observations and analysis:}

From the above calculations it is evident that efficiency of the HP heaters is on higher side kept for HPH-5. the efficiency of the HPH-5 may be lower due to sale formation or increase

flow velocity due to more number of tube diameters in the tube bundle. however efficiency can be improved by HP jet chemical cleaning of the tubes and replacement of the tube bundle the cycle efficiency of the plant can be improved.

\section{Extraction steam parameter HPH-5:}

Pressure of extraction steam $\mathrm{pr}=13 \mathrm{bar}$

Enthalpy of extraction steam hes $=3361.33 \mathrm{~kJ} / \mathrm{kg}$

Quantity of feed water flow Qf $=662 \mathrm{t} / \mathrm{hr}$

Temperature of feed water at inlet $\mathrm{Tfi}=169.3^{\circ} \mathrm{c}$

Temperature of feed water at outlet Tout $=186.0^{\circ} \mathrm{C}$

Quantity of extraction steam ( Qes) $=17.661 \mathrm{t} / \mathrm{hr}$

Quantity of drip from HPH-6 (Q d) $=202^{\circ} \mathrm{c}$

Enthalpy of drip from HPH-5 $=778.116 \mathrm{~kJ} / \mathrm{kg}$

Enthalpy of drip from HPH- $6=869.8 \mathrm{~kJ} / \mathrm{kg}$

Heat lost by extraction steam HPH-5 = Qe (hes - hfd)

$=17.661(3361.33-778.116) \times 10^{3}$

$=45622.14 \times 10^{3} \mathrm{~kJ} / \mathrm{kg}$

Heat lost by drip coming from HPH-6 = Qsd (hdri - hdrip)

$$
\begin{aligned}
& =79.719(869.8-778.116) \times 10^{3} \\
& =7308.96 \times 10^{3} \mathrm{~kJ} / \mathrm{kg}
\end{aligned}
$$

Total heat lost by extraction steam $\&$ drip:

$$
\begin{aligned}
& =52931.1 \times 10^{3} \mathrm{~kJ} / \mathrm{hr} \\
\text { Heat gained by feed water } & =\text { Qfdw }(\text { Tout }- \text { Tin }) \times 10^{3} \\
& =662 \times 4.1868(186-169.3) \times 10^{3} \\
& =46,286.75 \times 10^{3} \mathrm{~kJ} / \mathrm{hr}
\end{aligned}
$$$$
=(45622.4+7308.96) \times 10^{3}
$$

Efficiency of HPH - 5(n) $=4$ Heain

$=(46,286.75 / 52,931.1) \times 100$

$=87.45 \%$

\section{Extraction steam parameter HPH-6:}

Pressure of extraction steam $\mathrm{p} \mathrm{r}=28 \mathrm{bar}$

Enthalpy of extraction steam hes $=3071.72 \mathrm{~kJ} / \mathrm{kg}$

Quantity of feed water flow Qfw $=662 \mathrm{t} / \mathrm{hr}$

Temperature of feed water at inlet Tfi $=186^{\circ} \mathrm{c}$

Temperature of feed water at outlet Tout $=222.3{ }^{\circ} \mathrm{C}$

Quantity of extraction steam ( Qes) $=47278 \mathrm{~kg} / \mathrm{hr}$

Quantity of drip from HPH-7 (Q d) $=32441 \mathrm{t} / \mathrm{hr}$

Temperature of drip from HPH-6 $(\mathrm{Td})=202^{\circ} \mathrm{c}$

Temperature of drip from HPH-7 (Td) $=233^{\circ} \mathrm{c}$

Enthalpy of drip from HPH-6 $\mathrm{h} \mathrm{d}=869.8 \mathrm{KJ} / \mathrm{kg}$

Enthalpy of drip from HPH-7 $\mathrm{h} \mathrm{d}=1013.7 \mathrm{KJ} / \mathrm{kg}$

Heat lost by extraction steam HPH- $6=\mathrm{Q}_{\mathrm{es}}$ (hes - hfd)

$=47.278(3071.72-869.8) \times 10^{3}$

$=104102.37 \times 10^{3} \mathrm{~kJ} / \mathrm{kg}$

Heat lost by drip coming from HPH-7 = Qsd (hdri - hdrip) 
$=32441(1013.7-869.8) \times 10^{3}$

$=4668.26 \times 10^{3} \mathrm{~kJ} / \mathrm{kg}$

Total heat lost by extraction steam \& drip:

Heat gained by feed water $=$ Qfdw $($ Tout - Tin $) \times 10^{3}$

$$
\begin{aligned}
& =(104102.37+4668.26) \times 10^{3} \\
& =108770.63 \times 10^{3} \mathrm{~kJ} / \mathrm{hr}
\end{aligned}
$$

$$
\begin{aligned}
& =662 \times 4.1868(222.3-1876) \times 10^{3} \\
& =10041.37 \times 10^{3} \mathrm{~kJ} / \mathrm{hr}
\end{aligned}
$$

Efficiency of HPH - 6(n) $=$ Heat gained/heat lost

$=(100611.37 / 108770.63) \times 100$

$=92.5 \%$

Extraction steam parameter HPH-7:

pressure of extraction steam $\mathrm{p} \mathrm{r}=42 \mathrm{bar}$

enthalpy of extraction steam hes $=3168.06 \mathrm{~kJ} / \mathrm{kg}$

quantity of feed water flow Qfw $=662 \mathrm{t} / \mathrm{hr}$

Temperature of feed water at inlet Tfi $=222.3^{\circ} \mathrm{c}$

Temperature of feed water at outlet Tout $=245.7^{\circ} \mathrm{C}$

Quantity of extraction steam ( Qes) $=32441 \mathrm{~kg} / \mathrm{hr}$

Temperature of drip from HPH-7 (Td) $=233^{\circ} \mathrm{c}$

Enthalpy of drip from HPH-7 $\left(\mathrm{h}_{\mathrm{Fd}}\right)=1013.7 \mathrm{~kJ} / \mathrm{kg}$

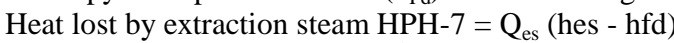

$=37441(3168.06-1013.7) \times 10^{3}$

$=69889.6 \times 10^{3} \mathrm{~kJ} / \mathrm{kg}$

Heat gained by feed water $=\mathrm{Qfdw}($ Tout - Tin $) \times 10^{3}$

$$
=662 \times 4.1868(245.7-222.3) \times 10^{3}
$$

Efficiency of HPH - 7 = Heat gained/heat lost

$$
=64856.88 \times 10^{3} \mathrm{~kJ} / \mathrm{hr}
$$

$=(64856.88 / 69889.6) \times 100$

$=92.8 \%$

Total efficiency of the turbine $($ HP,IP,LP $)=((87.45+92.5+92.8) / 3) \times 100$

$=(272.75 / 3) \times 100$

$=90.91 \%$

\section{Conclusion:}

The efficiency of power plant increase with increase in number of heaters, but it is not economical to have large number of heaters to increase to increase the efficiency on the basis of the techno-economic study the numbers of heaters generally used in $210 \mathrm{MW}$ units are 6 to 7 thus the efficiency increases by 5 to 6 \%approximately.The operation and maintenance of the heaters in the current power plant must be increased such that there is no much deviation in the design and calculations values. It is also observed that with this operation and maintenance the heaters are giving the efficiency of about $75 \%-80 \%$.

\section{Examples follow:}

\section{References}

\section{Journal Papers:}

[1]. Drbal..westra K. and boston P...1995.power plant Engineering.springer

[2]. Szargut J., 2004, Economic effect of the regenerative heating of feed water in a steam power plant (in polish).energetyka no.5, pp. $266-268$

[3]. Szargut J., 1999, Application of steam from regenerative bleeds for the production of network in large steam power Books: plants.ArchiwumEnergetvki.XXVIII, No.1-2.pp.83-93

[4]. R.k raj put,ThermalEgg,Laxmipublication (P) LTD (ISBN-978-81-318-0804-7), eighth Edition,andP.no:644

[5]. P.K nag,Egg Thermodynamic, McGraw-hill (ISSBN-10:0-07-026062-1),Fourth Edition,P.no:457,532,449

[6]. AroraDomkundwar, power plant Engg,Dhanpatrai\&co.(P) LTD,First Edition,P.no:19.1-19.11,22.1-22.70

[7]. R.SKhurmi\&J.KGupta,ThermalEngg,S.chanpublishing(ISBN: 81-219-2573-8),FirstEditon,P.no:569,573.

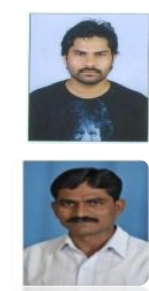

CH.vijaya kumarM.Tech (CAD/CAM), Asst.Professor in Guru Nanak Inst Of Technology, Ibrahimpatnam, R.R Dist-501506,

Emid:vijaya_kumar580@yahoo.com, Cell:9652898481.

Dhananjaya kumar M.E, Asso.pofessor in GNIT.

Emid:drawookar@yahoo.co.in,cell:08050565245 